Mol. Cryst. Liq. Cryst., 1997, Vol. 304, pp. 363-370

Reprints available directly from the publisher

Photocopying permitted by license only
(1997 OPA (Overseas Publishers Association) Amsterdam B.V. Published in The Netherlands under license by Gordon and Breach Science Publishers

\title{
MATRIX ADDRESSING WAVEFORMS FOR GREY SHADES SSFLC
} DISPLAYS

V. FERRARA, R. BECCHERELLI, F. CAMPOLI, A. D'ALESSANDRO, A. GALLOPPA, A. GALBATO, P. MALTESE

Department of Electronic Engineering, La Sapienza University and National Institute of Physics of Matter, Rome, Italy.

\begin{abstract}
To produce analog grey shades in our special construction SSFLC matrix display, different "low voltage", "stopped writing" and "hampered writing" addressing waveforms have been tested in computer simulations and experiments. Three phenomena limit the achievable results: the first is addressing hysteresis, subject of our previous paper, the second is the crosstalk from data intended for other pixels, the third is characteristic switching pulse area variations due to cell temperature or thickness variations. We present here computer simulations of the two latter effects, for a range of different addressing modes. For the faster addressing modes, based on liquid crystal dielectric biaxiality, data waveforms are necessary featuring constant rms voltage, dc balance and crosstalk compensation. New technique are here introduced making use of such data waveforms in place of the previous ones, based on amplitude or pulse-position modulation.
\end{abstract}

\section{INTRODUCTION}

Recently several fast and high voltage addressing modes for SSFLC displays have been presented. Control window is placed either between the end of the writing pulse and the beginning of the stop pulse ("Superfast") ${ }^{1}$ or at the beginning of the writing pulse ("Malvern") ${ }^{2}$. In the former case lower selection voltages in combination with higher spontaneous polarization $\left(\mathrm{P}_{s}\right)$ liquid crystals giving optically better stable states can be used, whereas in the latter one wider operating regions with lower data voltages can be obtained.

Improved optical performances and increased operating regions in the V-t plane can be obtained with the control window around a callback pulse and using a compensation pulses in the selection waveform and crosstalk compensated data waveforms ("Rome" and "Compensated Rome" modes). ${ }^{3}$

We present here the results of several simulations on these modes employing measured cell parameters, ${ }^{4}$ in which their ranges of operation are characterized and compared. 
We also tested these modes with data modulation techniques in order to obtain grey shades in matrix addressed displays. It is worth noting that, for "High voltage" addressing, data waveforms must fulfil three fundamental conditions: a constant rms voltage, dc balance and crosstalk compensation. ${ }^{5}$ The AM modulation technique does not fulfil the first condition and it is more difficult to implement in fast high voltage IC drivers. Two levels pulse position modulation (PPM) is easier to implement but it provides crosstalk compensation only with extreme data. Thus we propose new grey shade addressing methods fullfilling all three requirements.

We finally present experimental results on grey scale matrix addressing.

\section{ADDRESSING MODES}

The "low voltage" addressing modes have been explained in terms of a critical pulse area. Voltage-square dependent dielectric torques are exploited in the faster "high voltage" modes. At rather high voltages such torques succeed in modifying switching due to the linear dependent voltage polarization torques.

"High voltage" modes have been classed in "Hampered Writing" and "Stopped Writing" modes, with the control window placed respectively either at the beginning or at the end of the switching process. ${ }^{6}$ The former are based on dielectric retarding effects in the initial stage of switching, whereas the latter are based on the accelerating effects in the final stage. "Hampered Writing" modes are the least sensitive to the variations in the switching pulse area.

Our recent investigations deal with "Compensated Rome" modes working at lower voltages, higher contrast, higher speed and featuring compensation versus temperature and cell thickness variations. ${ }^{5,9}$

Figure 1 shows a typical example of such matrix addressing waveforms which use crosstalk compensated data. The complex time-overlapping dc-balanced pulse sequence used for selection includes two erasing pulses, one compensation pulse for switching pulse area variations, a first writing pulse, one callback pulse and a second writing pulse.

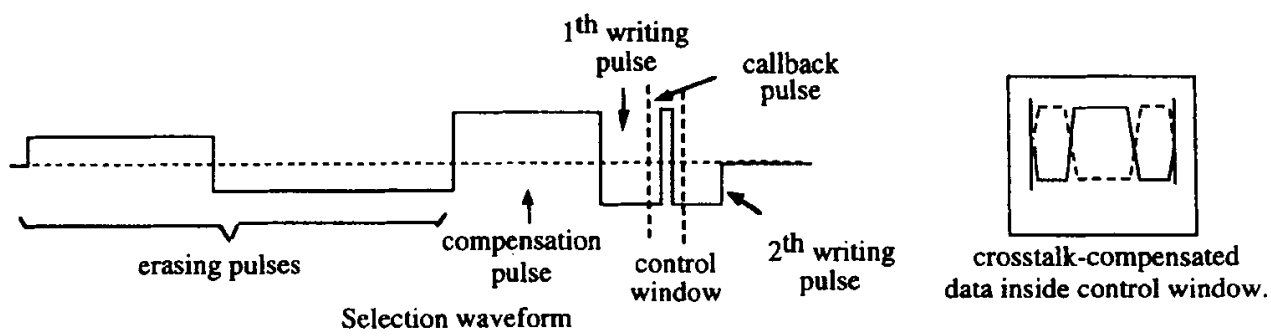

FIGURE 1 "Compensated Rome" addressing mode scheme. 
Only data inside the control window which includes the callback pulse and part of writing pulses are ideally active. For maximum control effect, inside the control window, both the selection and data waveforms should be dc-balanced and change sign at the same time. Data waveforms shown in Figure 1 are for black and white. Intermediate waveforms must be provided to obtain grey shades and they should be dc-balanced and crosstalk compensated too. Use of non-crosstalk-compensated data would cause large sensitivity of the response to the data preceding and following the one in the control window.

\section{COMPUTER SIMULATION}

We have embedded our simple uniform director model of SSFLC cells subject to matrix addressing waveforms ${ }^{7}$, in two interactive software programs 8 : the former to design or check new addressing schemes, the latter to calculate their operating ranges.

By computer simulation we have investigated the operating regions of many "Compensated Rome" modes differing each other for the pulse durations, as shown in Table 1, and for the selection/data voltage amplitude ratios. Cell parameters previously identified ${ }^{4}$ for typical Merck ZLI 4655-000 and BDH SCE8 $1.7 \mu \mathrm{m}$ cells at $30^{\circ} \mathrm{C}$ have been employed.

TABLE I "Compensated Rome" modes - Length of selection waveform pulses normalized to line addressing time.

\begin{tabular}{l|cccccc}
\hline & \multicolumn{6}{|c}{ Pulses } \\
\hline Mode & $\begin{array}{c}\text { 1st } \\
\text { erasing }\end{array}$ & $\begin{array}{c}\text { 2nd } \\
\text { erasing }\end{array}$ & Compensation & $\begin{array}{c}\text { 1st } \\
\text { writing }\end{array}$ & callback & $\begin{array}{c}\text { 2nd } \\
\text { writing }\end{array}$ \\
\hline CROM4 & 4 & 6 & 4 & 1.25 & 0.5 & 1.25 \\
CROM5 & 5 & 7 & 4.5 & 1.75 & 0.5 & 1.25 \\
CROM6 & 6 & 8 & 5 & 2.25 & 0.5 & 1.25 \\
CROM7 & 7 & 9 & 5.5 & 2.25 & 0.5 & 1.75 \\
CROM8 & 8 & 10 & 6 & 2.25 & 0.5 & 2.25
\end{tabular}

In Figure 2 the operating regions are shown of several "Compensated Rome" modes as computed for the SCE8 cell, with an additional limitations on maximum data amplitude producing alignment defects. All the boundaries in the $V-t$ plane correspond to worst case data patterns calculated in every point. Operation in different $V$ - $t$ regions can be obtained by choosing the pulse lengths as described in Table 1 . The proper selection to data voltage ratios are then chosen in order to give the widest operating region. The similar Figure 3 
corresponds to the simulation for a cell filled with ZLI 4655-000 and maximum amplitude of data voltage equal to $25 \mathrm{~V}$.

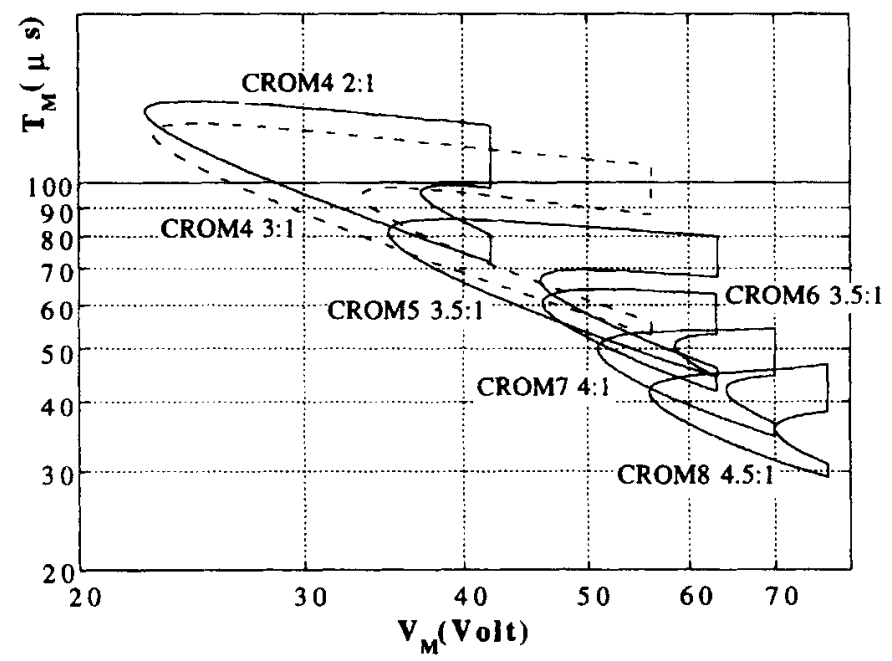

FIGURE 2 Computed regions of operation of addressing modes for our model of a SCE8 cell at $30^{\circ} \mathrm{C}$ (Ref. 4) in a line addressing time - total voltage plane $T_{M}-V_{M}$. Selection/data ratios are indicated. For every mode the boundary is limited at the right side by the maximum amplitude of data voltage (14 V) which does not damage the cell.

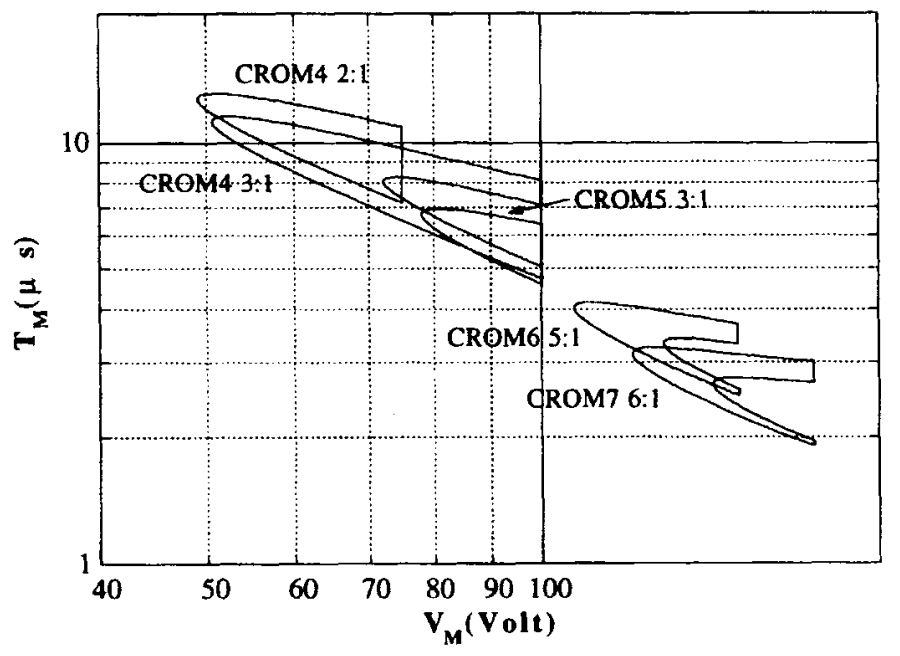

FIGURE 3 Computed regions of operation of addressing modes for our model of a ZLI 4655-000 cell at $30^{\circ} \mathrm{C}$ (Ref. 4). In this case the boundary is limited at the right side by a data voltage equal to $25 \mathrm{~V}$. 


\section{ADDRESSING MODES FOR GREY SHADES SSFLC DISPLAY}

In the development of Switching Area Control (SAC) display, which allows a virtually analog grey scale ${ }^{9}$, besides reducing undesired effects (i.e. hysteresis and image sticking), we experimented grey scale implementation of the most effective addressing modes. Many experiments have been accomplished using "Compensated Rome" modes with Pulse Position Modulation (PPM) data techniques in order to obtain as much as possible distinguishable, reproducible and stable intermediate levels ${ }^{9}$. In a better solution, the intermediate waveforms are modified, as shown in Fig. 4a, by adding a pulse, so that also the intermediate waveforms are crosstalk-compensated. Time fraction $\alpha=t_{1} / T_{\text {addr }}$ and $\gamma=\mathrm{t}_{3} / \mathrm{T}_{\text {addr }}$ can be computed as functions of $\beta=\mathrm{t}_{2} / T_{\text {addr }}$ as:

$$
\begin{aligned}
& \alpha=\frac{1+2 \beta-\sqrt{4 \beta^{2}-4 \beta+3}}{4} \\
& \gamma=\frac{1+2 \beta+\sqrt{4 \beta^{2}-4 \beta+3}}{4}
\end{aligned}
$$

In practice, it turns out difficult to subdivide the control windows in non-regular time intervals. With this additional requirement, approximate crosstalk-compensation can still be obtained by means of sets of data waveforms, as the one shown as an example, for 16 time subintervals, in Figure $4 \mathrm{~b}$.

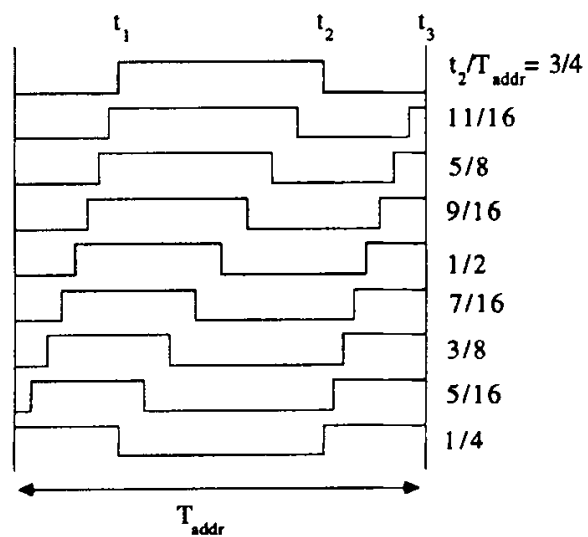

a)

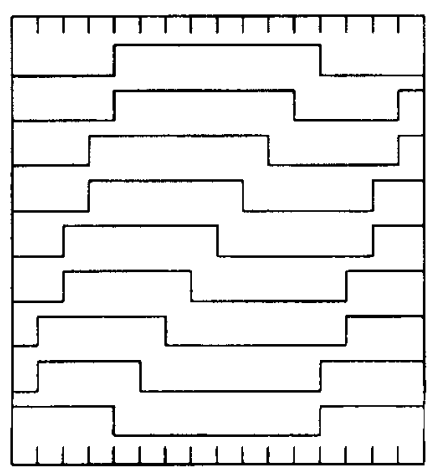

b)

FIGURE 4. (a) A possible choice of crosstalk compensated data waveforms computed according (1), (2) and (b) their approximate practical implementation.

For all data waveforms shown, the number of shades is limited by the number of available intermediate times in the control window. We have found that, for a given number of intermediate times, similar results can be obtained with a completely different 
technique, shown in Figure 5. Eight data voltages in the set, corresponding to eight grey levels, interact with a selection waveform whose final part only is drawn. Four different waveforms, in their direct and inverted versions, provide dc balance and crosstalkcompensation, also presenting null cross-correlation integrals.
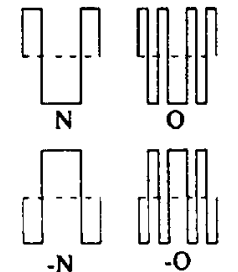
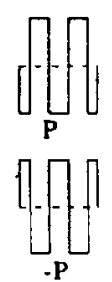

a)

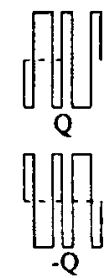

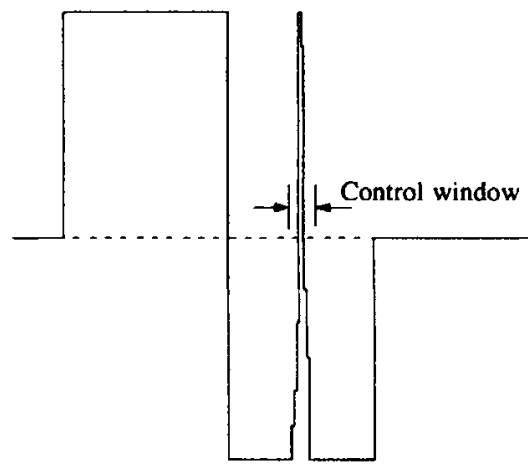

b)

FIGURE 5. (a) dc balanced, crosstalk-compensated data waveforms; (b) final part of selection waveform (erasing pulses not shown).

Control effects are proportional to the correlation between selection and data voltages in the control window. 5 To achieve control effects proportional to assigned weights, the selection voltage in the control window has been properly shaped. To get equidistant gradations, weights 7, 5, 3 and 1 have been chosen respectively for $\mathrm{N}, \mathrm{O}, \mathrm{P}$, and $Q$ data. By so doing the selection voltage can be shaped as a single callback pulse with eight voltage steps. The selection sequence shown is preceded by erasing pulses, that also balance its dc component.

In Figure 6 calculated optical transmissions are given for the addressing scheme of Figure 5 and a simulated SCE4 cell. Eight different data waveforms produce eight equidistant levels in accordance to the previously described design.

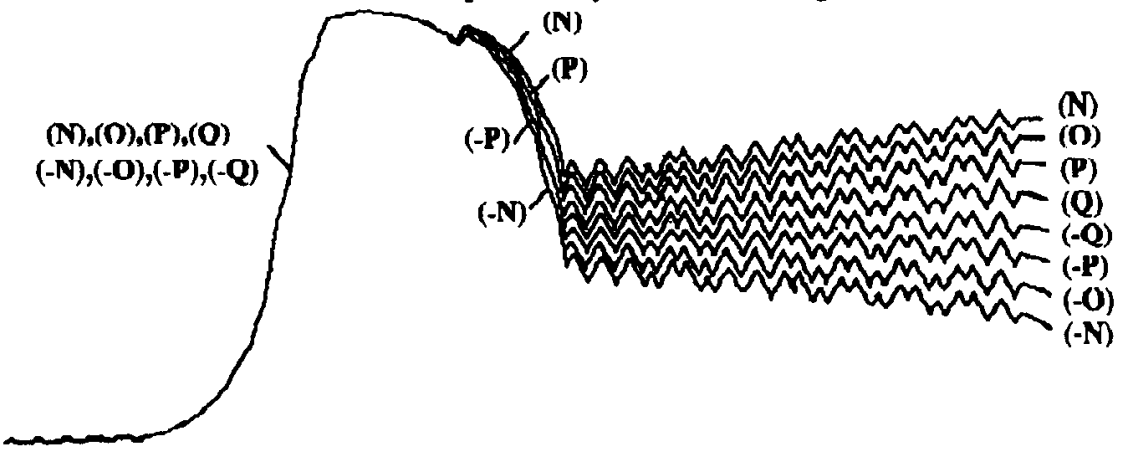

FIGURE 6 Optical transmission for a simulated SCE4 cell employing $40 \mathrm{~V}$ and $13 \mathrm{~V}$ for selection and data voltage respectively and $36 \mu \mathrm{s}$ as addressing time. 


\section{CELLMEASUREMENTS}

The most promising simulated addressing modes have been experimentally verified with a special experimental setup ${ }^{10}$ giving a good agreement with simulation result. In order not to stress the cells we performed measurements only with voltages as low as possible.

In Figure 7 a fotomultiplier output voltage corresponding to the optical transmission of a SAC cell filledwith SCE8 and aligned with teflon layers ${ }^{11}$ is reported, as recorded by a digital oscilloscope. The cell was placed between crossed polarizer adjusted for symmetrical response between black and white and not for maximum contrast. Cell was addressed with a CROM7 mode with $36 \mathrm{~V}$ peak selection amplitude. Quasi-crosstalkcompensated PPM data (as in Figure $4 \mathrm{~b}$ ) with $12 \mathrm{~V}$ peak have been applied to all the columns in order to obtain progressive and uniform switching area control from metal tracks to the center of pixels. A $48 \mu$ s line addressing time and a uniform crosstalk compensated data background corresponding to the black state was used. For some levels the stabilization effect toward stable states performed by high frequency data is clearly seen. Good grey scale uniformity and reproducibility were obtained.

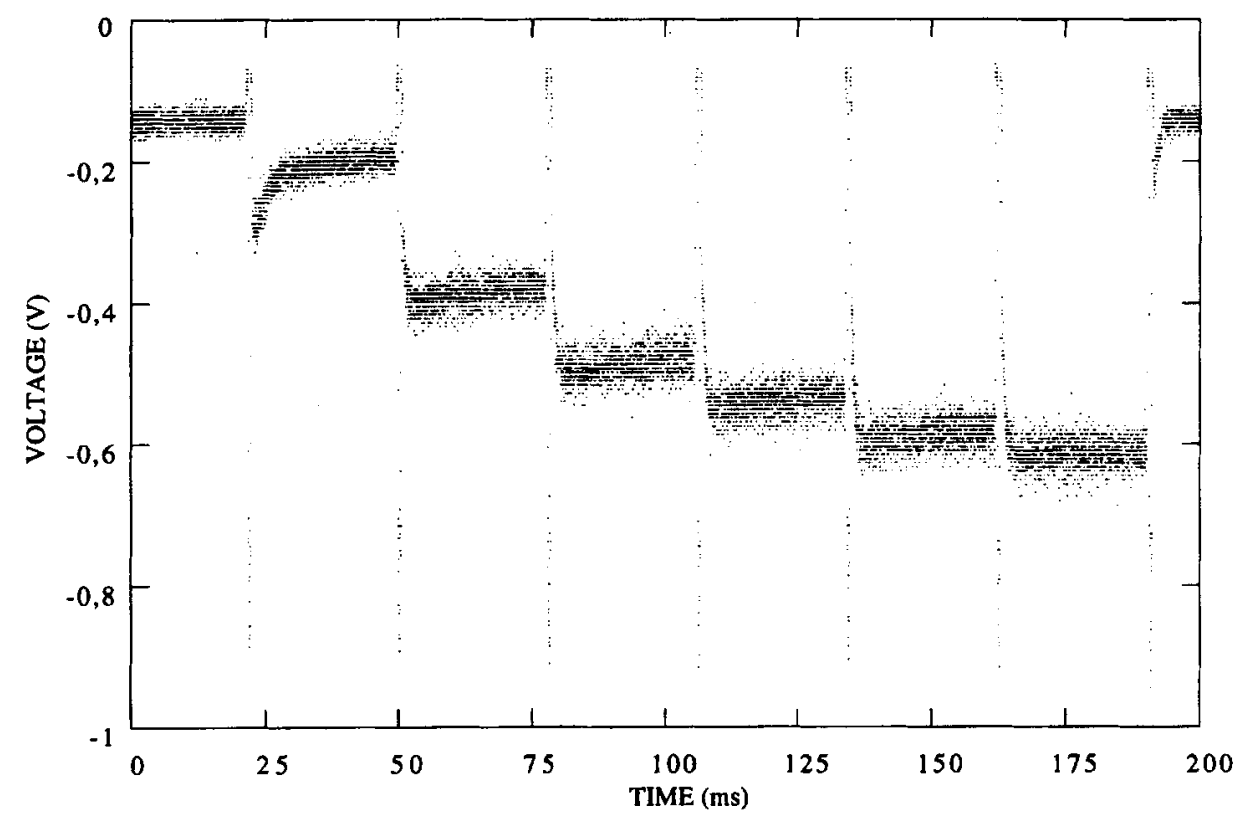

FIGURE 7 Fotomultiplier output voltage sampled by a digital oscilloscope corresponding to the optical transmission integrated over pixel area of a SAC cell filled with SCE8. Less negative voltage corresponds to black state and spikes to multiple pulse selection waveform. 


\section{CONCLUSIONS}

We presented a comparative study of computed operating regions of "Compensated Rome" modes using measured parameter of two cells filled with low spontaneous polarization liquid crystals. Wide operating regions are obtained with physically applicable voltages.

We proposed innovative data modulation techniques able to give gradation control while avoiding undesired crosstalk effects.

Finally experimental results validating simulation on a Switching Area Control display cell were shown .

\section{REFERENCES}

1. P. Maltese, R. Piccolo, Intl. SID Symposium, p. 642 (1993).

2. J. R. Hughes and E. P. Raynes, Liquid Crystals, 13 p. 597 (1993) and errata corrige 15 , p. 281 (1993).

3. P. Maltese, V. Ferrara, International Display Research Conference, Monterey, California, p. 277 (Oct. 1994).

4. T. Matuszczyk, M. Buivydas, S.T. Lagerwall, F. Bernardini, P. Maltese, International Display Research Conference, Monterey, California, p. 221 (Oct. 1994).

5. P. Maltese, V. Ferrara, Journal of the SID 4, N. 2 (1996).

6. P. Maltese, V. Ferrara, A. Concettini, Mol. Cryst. Liq. Cryst., Vol. 266, p. 163 (1995).

7. P. Maltese, R. Piccolo e V. Ferrara, Liquid Crystal, 15, No. 6, p. 819 (1993).

8. P. Maltese, V. Ferrara, R. Piccolo, SID symposium, San Josè, California, p. 151 (June 1994).

9. P. Maltese, F. Campoli, A. d'Alessandro, V. Foglietti, A. Galbato, A. Galloppa, G. Rafaelli and M. Wnek, Eerroelectrics. 179, 153 (1996).

10. P. Maltese, R. Beccherelli, F. Bernardini, M. Wnek and F. Zuliani, Ferroelectrics, Vol. 178, p. 27 (1996)

11. F. Campoli, R. Beccherelli, A. d'Alessandro, V. Ferrara, A. Galloppa, G.Padeletti, S. Petrocco, P. Maltese, presented at ILCC96 and submitted to $\underline{\text { Mol. }}$ Cryst. Liq. Cryst. 